\title{
Tratamiento adyuvante de melanoma cutáneo: Indicaciones y alternativas
}

\section{Adjuvant Treatment of Cutaneous Melanoma - Indications and Alternatives}

\author{
" Mauricio Lema Medina MD Esp1 2* \\ »Camila Lema Calidonio MD MSc1. \\ 'Departamento Hematología y Oncología, Clínica de Oncología Astorga. Medellín -Colombia. \\ 2 Clínica SOMA. Medellín - Colombia.
}

Recibido el 28 de junio de 2020; Aceptado el 28 de julio de 2020

\section{Resumen}

La cirugía es la principal modalidad de tratamiento para el melanoma no metastásico, y el melanoma metastásico resecable. Sin embargo, la supervivencia libre de enfermedad (SLE) a 10 años después de cirugía en melanoma estadio III se estima de $24 \%$ a $88 \%$. Diversos estudios han explorado la opción de terapia adyuvante, incluyendo interferón, inmunoterapia y terapia dirigida con agentes anti BRAF/MEK en pacientes con mutación de BRAF. En este artículo se revisa la evidencia relevante de terapias sistémicas adyuvantes en melanoma cutáneo. El interferón alfa de altas dosis demostró un modesto beneficio en SLE, a expensas de elevada toxicidad y ya no se usa en Colombia. De igual forma, la inmunoterapia con ipilimumab es menos eficaz y más tóxica que la inmunoterapia con nivolumab, un anticuerpo monoclonal anti muerte programada 1 (anti-PD1) en un estudio que incluyó pacientes con estadios IIIB-IV luego de resección completa. Pembrolizumab, otro anti-PD1, también es eficaz después de resección en melanoma estadio III (con depósito tumoral ganglionar $>1 \mathrm{~mm}$ ). Para pacientes con mutación de BRAF V600, la terapia dirigida oral con dabrafenib más trametinib por un año es una alternativa para estadio III (con depósito tumoral ganglionar $>1 \mathrm{~mm}$ ). La selección de la terapia a administrar depende de factores clínicos como estadio y moleculares. En general, se recomienda alguna forma de terapia adyuvante con anti-PD1 o terapia dirigida en melanoma resecados con alto riesgo de recaída.

Palabras clave: Melanoma; quimioterapia adyuvante; sobrevida.

\section{Abstract}

Surgery is the main treatment modality for non-metastatic melanoma, and resectable metastatic melanoma. However, 10-year disease-free survival (DFS) after surgery for stage III melanoma ranges from $24 \%$ to $88 \%$. Several studies have explored the option of adjuvant therapy, including interferon, immunotherapy, and targeted therapy with anti-BRAF / MEK agents in patients with a BRAF mutation. This article reviews the relevant evidence for adjuvant systemic therapies in cutaneous melanoma. High-dose interferon alpha demonstrated modest benefit in SLE, at the expense of high toxicity, and is no longer used in Colombia. Similarly, immunotherapy with ipilimumab is less effective and more toxic than immunotherapy with nivolumab, an anti-programmed death 1 (anti-PD1)

\footnotetext{
* Autor para correspondencia: Mauricio Lema Medina. MD, Hemato oncólogo. Departamento Hematología y Oncología Clínica de Oncología Astorga y Clínica SOMA. Correo electrónico: mauriciolema@yahoo.com

Sociedad Colombiana de Hematología y Oncología. Este es un artículo Artículo en Acceso Abierto. Licencia Creative Commons Reconocimiento-NoComercial-SinObraDerivada (https://creativecommons.org/licenses/by-nc-nd/4.0/)
} 
monoclonal antibody in a study that included patients with stage IIIB-IV after complete resection. Pembrolizumab, another anti-PD1, is also effective after resection in stage III melanoma (with nodal tumor deposit $>1 \mathrm{~mm}$ ). For patients with a BRAF V600 mutation, oral targeted therapy with dabrafenib plus trametinib for one year is an alternative for stage III (with nodal tumor deposit $>1 \mathrm{~mm}$ ). The treatment selection to be administered depends on clinical factors such as stage and molecular. In general, some form of adjuvant anti-PD1 therapy or targeted therapy is recommended in resected melanoma at high risk of relapse.

Keywords: Melanoma; chemotherapy adjuvant; survival.

\section{Introducción}

El melanoma cutáneo estadio III se caracteriza por compromiso de ganglios regionales, y se asocia a supervivencia melanoma-específica a diez años, que oscila entre $24 \%$ y $88 \%{ }^{1}$. El riesgo alto de recidiva y muerte en este subgrupo de pacientes, es el ímpetu que ha guiado a más de 20 años de investigación, el papel de la terapia sistémica adyuvante. En este artículo se discuten las indicaciones y alternativas disponibles para melanoma cutáneo. La Tabla 1 resume los hallazgos de los diferentes estudios en mención.

\section{La etapa III en la octava clasificación de melanoma del Comité Conjunto Estadounidense de Cáncer (AJCC)}

En la octava clasificación de la AJCC de melanoma se hizo una revisión sustancial de las definiciones de etapa III, para demarcar mejor la extraordinaria heterogeneidad que existe entre sus distintos subgrupos. La supervivencia melanoma-específica a diez años de $88 \%, 77 \%$, $60 \%$ y $24 \%$ para estadios IIIA, IIIB, IIIC, y IIID, respectivamente ${ }^{1}$. Sin embargo, los estudios que se analizan, fueron realizados con clasificaciones que no tenían el grado de granularidad presente. Por ejemplo, la categoría IIID es nueva, y no aparece en los criterios de inclusión de los estudios en su publicación original. En este documento se reflejan las etapas en los criterios de inclusión y exclusión como si los estudios se hubieran realizado en la era actual.

\section{Interferón alfa}

En la última década del siglo 20 se publicaron varios estudios con interferón alfa en ambos lados del Atlántico. Los estudios norteamericanos fueron realizados por el Grupo Cooperativo de Oncología del Este (ECOG, por sus siglas en inglés) con los estudios E1684, E1690 y E1694, que exploraron el beneficio de la administración de altas dosis de interferón alfa ${ }^{2-5}$.

En Europa se realizó el estudio EORTC 18991, que exploró la utilización de interferón alfa peguilado por varios años contra observación ${ }^{6,7}$. Tanto en los estudios de la ECOG como de la EORTC se incluyeron pacientes con compromiso ganglionar regional. Una particularidad especial de los estudios de la ECOG, es que también incluyeron pacientes T4 No (etapas IIB y IIC de la clasificación actual), y se constituyen en los únicos estudios en incluir este subgrupo de pacientes. La adición de interferón adyuvante mejora la supervivencia libre de recaída, con un impacto modesto en la supervivencia general. En un metaanálisis que incluyó más de 17 estudios con 10.345 pacientes, se concluye que la adyuvancia con interferón se asocia a una reducción de riesgo relativo del $9 \%{ }^{8}$. El beneficio en la supervivencia libre de recaída con altas dosis de interferón se mantiene luego de 16 años de seguimiento mediano ${ }^{9}$.

Los autores constatan en esa misma publicación que la supervivencia general en el análisis agrupado de los estudios E1684 y E1690, se desvaneció.

El modesto impacto del interferón en la supervivencia general, así como su elevada toxicidad y costo, se reflejaron en su baja adopción 
Tabla 1. Estudios modernos de terapia sistémica adyuvante en melanoma cutáneo.

\begin{tabular}{|c|c|c|c|c|c|c|c|c|}
\hline & \multicolumn{2}{|c|}{ EORTC 18071} & \multicolumn{2}{|c|}{ CheckMate-238 } & \multicolumn{2}{|c|}{ KEYNOTE 054} & \multicolumn{2}{|c|}{ COMBI-AD } \\
\hline $\begin{array}{l}\text { Número de } \\
\text { pacientes } \\
\text { incluidos }\end{array}$ & \multicolumn{2}{|c|}{951} & \multicolumn{2}{|c|}{906} & \multicolumn{2}{|c|}{1019} & \multicolumn{2}{|c|}{870} \\
\hline Estadios & \multicolumn{2}{|l|}{ III } & \multicolumn{2}{|c|}{$\begin{array}{l}\text { III B-C } \\
\text { IV }\end{array}$} & \multicolumn{2}{|c|}{ III A-C } & \multicolumn{2}{|c|}{$\begin{array}{c}\text { III A-C } \\
\text { BRAF mutado }\end{array}$} \\
\hline $\begin{array}{l}\text { Brazos de } \\
\text { tratamiento }\end{array}$ & $\begin{array}{c}\text { ipilimumab* } \\
* \\
(n=475)\end{array}$ & $\begin{array}{c}\begin{array}{c}\text { placebo* } \\
* \\
(n==476)\end{array}\end{array}$ & $\begin{array}{l}\text { nivolumab } \\
\text { (n=453) }\end{array}$ & $\begin{array}{l}\text { ipilimumab } \\
\text { (n=453) }\end{array}$ & $\begin{array}{c}\text { pembrolizuma } \\
b \\
(n=514)\end{array}$ & $\begin{array}{l}\text { placebo } \\
(n=505)\end{array}$ & $\begin{array}{c}\text { dabrafenib + } \\
\text { trametinib } \\
(n=438)\end{array}$ & $\begin{array}{l}\text { placebo } \\
(n=432)\end{array}$ \\
\hline $\begin{array}{l}\text { Supervivencia } \\
\text { libre de } \\
\text { recurrencia }\end{array}$ & $46.5 \%$ & $34.8 \%$ & $70.5 \%$ & $60.8 \%$ & $75.4 \%$ & $61.0 \%$ & $58.0 \%$ & $39.0 \%$ \\
\hline $\begin{array}{l}\text { Tiempo de } \\
\text { seguimiento }\end{array}$ & \multicolumn{2}{|c|}{3 años } & \multicolumn{2}{|c|}{12 meses } & \multicolumn{2}{|c|}{12 meses } & \multicolumn{2}{|c|}{3 años } \\
\hline $\mathrm{HR}^{*}$ & \multicolumn{2}{|c|}{$\begin{array}{c}0.75(\mathrm{IC} 95 \%: \\
0.64-0.90 ; P<0.001)\end{array}$} & \multicolumn{2}{|c|}{$\begin{array}{c}0.65 \text { (IC } 97.56 \%: 0.51-0.83 \\
\text { P }<0.001)\end{array}$} & \multicolumn{2}{|c|}{$\begin{array}{c}0.57 \text { (IC } 98.4 \%: 0.43-0.74 \\
P<0.001)\end{array}$} & \multicolumn{2}{|c|}{$\begin{array}{c}0.47 \% \text { (IC } 95 \%: 0.39-0.58 ; \\
P<0.001)\end{array}$} \\
\hline $\begin{array}{l}\text { Porcentaje de } \\
\text { descontinuación } \\
\text { por toxicidad }\end{array}$ & $49.0 \%$ & $4.0 \%$ & $3.5 \%$ & $30.0 \%$ & $13.0 \%$ & $1.6 \%$ & $26.0 \%$ & $3.0 \%$ \\
\hline $\begin{array}{l}\text { Toxicidades } \\
\text { grado } 3 / 4\end{array}$ & $54.0 \%$ & $25.0 \%$ & $25.4 \%$ & $55.2 \%$ & $31.6 \%$ & $18.5 \%$ & $41.0 \%$ & $14.0 \%$ \\
\hline
\end{tabular}

por la comunidad oncológica, como se evidencia con placebo como brazo control de muchos de los estudios subsecuentes.

Ninguno de los interferones discutidos en este apartado están disponibles actualmente en Colombia.

\section{Ipilimumab}

La activación de los linfocitos T citotóxicos por intermedio de las células presentadoras de antígeno se puede restablecer con ipilimumab, un anti-CTLA4. La eficacia de la inmunoterapia con ipilimumab se estableció en melanoma metastásico en $2010^{10}$. El paso lógico siguiente fue explorar el beneficio potencial de ipilimumab adyuvante. El estudio EORTC 18071 comparó altas dosis de ipilimumab contra placebo en pacientes con melanoma resecado etapa III ${ }^{11}$. Para ser incluidos en el estudio, los pacientes debían tener una resección completa del melanoma con márgenes adecuados del tumor primario y, además, se requería la realización de vaciamiento ganglionar regional. Se incluyeron 951 pacientes con melanoma cutáneo etapa III (se excluyeron pacientes con metástasis ganglionar de $\leq 1 \mathrm{~mm}$, o con metástasis en tránsito). El brazo experimental consistió en ipilimumab 10 $\mathrm{mg} / \mathrm{kg}$ cada 21 días, seguido por la misma dosis cada tres meses, hasta por tres años. El brazo control recibió placebo. Después de cinco años de seguimiento, la supervivencia libre de recurrencia fue de $40.8 \%$ en el grupo de ipilimumab, comparado con $30.3 \%$ en el grupo placebo. Lo que constituyó una reducción del $24 \%$ en la probabilidad de recurrencia para el brazo con ipilimumab. La supervivencia general a cinco años fue también superior con ipilimumab $(65.4 \%$ en 
el grupo de ipilimumab comparada con $54.4 \%$ en el grupo placebo), así como la supervivencia libre de metástasis (48.3\% en el grupo de ipilimumab, comparado con $38.9 \%$ en el grupo placebo) ${ }^{11}$.

Los eventos adversos grado 3 o 4 ocurrieron en $54.1 \%$ de los pacientes con ipilimumab, comparado con $26.2 \%$ en el grupo placebo. Las toxicidades inmuno-relacionadas fueron también mayores en el grupo de ipilimumab (41.6\%, comparadas con $2.7 \%$ en el grupo placebo). Cinco pacientes ( $1.1 \%$ ) en el brazo de ipilimumab fallecieron por eventos adversos inmunomediados ${ }^{11}$.

En octubre de 2015 la Agencia de Alimentos y Medicamentos de los Estados Unidos (FDA, por sus siglas en inglés) aprobó adyuvancia con ipilimumab en dosis altas para pacientes con melanoma etapa III.

El estudio E1609 demostró que la supervivencia libre de recaída fue esencialmente idéntica al comparar ipilimumab $10 \mathrm{mg} / \mathrm{kg}$ contra $3 \mathrm{mg} / \mathrm{kg}$, con una reducción modesta en la toxicidad ${ }^{12}$.

\section{Inhibidores de PD1}

Los agentes anti PD1 nivolumab y pembrolizumab se convirtieron en un estándar de tratamiento para pacientes con melanoma avanzado. Estos agentes causan la activación de los linfocitos T efectores, al bloquear retenes inmunológicos que involucran PD1. Tanto el nivolumab como el pembrolizumab demuestran mejor supervivencia general al compararlos con dacarbazina e ipilimumab, respectivamente ${ }^{13,14}$. Como ocurrió con ipilimumab, tanto nivolumab como pembrolizumab han sido estudiados en el entorno adyuvante.

En el estudio CheckMate-238 se incluyeron 906 pacientes con melanoma y resección completa de alto riesgo ${ }^{15}$. El brazo experimental consistió en nivolumab $3 \mathrm{mg} / \mathrm{kg}$ intravenoso, cada dos semanas, por un año. El brazo control fue ipilimumab $10 \mathrm{mg} / \mathrm{kg}$. El desenlace principal fue la supervivencia libre de recaída. Se incluyeron pacientes: $\geq 15$ años de edad, desempeño ECOG o/1, etapas IIIB-D resecados. Se requirió linfadenectomía regional completa o resección dentro de 12 semanas antes de la aleatorización. Tam- bién se incluyeron pacientes metástasis (etapa IV) resecados, incluyendo metástasis del sistema nervioso central. Los principales criterios de exclusión incluyeron: melanoma ocular o uveal, historia de enfermedad autoinmune, antecedente de otra neoplasia maligna sin remisión completa por los últimos tres años antes de la aleatorización, requerimiento de uso continuo de glucocorticoide sistémico, y antecedente de terapia sistémica previa para el melanoma ${ }^{15}$.

Luego de un seguimiento mediano de 18.5 meses se documentó la superioridad del nivolumab sobre el ipilimumab, con un cociente de riesgo de 0.65 (supervivencia libre de recaída a los 12 meses de $70.5 \%$ vs. $60.8 \%$, a favor de nivolumab). La superioridad de nivolumab fue independiente de la expresión de PD-L1 o de mutación del BRAF en las células tumorales. El beneficio de nivolumab sobre ipilimumab se mantuvo con el seguimiento a 36 meses (58\% comparado con $45 \%$, a favor de nivolumab) ${ }^{16}$. Cabe anotar que el CheckMate-238 es único en la literatura adyuvante en melanoma, que ha incluido pacientes con enfermedad metastásica (etapa IV) con resección completa.

Las toxicidades grado 3/4 fueron mayores en el brazo de ipilimumab al compararlo con nivolumab ( $55.2 \%$ y $25.4 \%$, respectivamente). Las toxicidades más comunes con nivolumab incluyeron: fatiga, diarrea, prurito, exantema, náuseas, artralgias e hipotiroidismo. Los eventos adversos con ipilimumab fueron similares, pero en mayor proporción, particularmente la diarrea. La descontinuación del tratamiento por eventos adversos ocurrió en 3.5\% de los pacientes del brazo de nivolumab, comparado con $30 \%$ en el de ipilimumab ${ }^{15}$.

En diciembre de 2017 se aprobó nivolumab por la FDA en los Estados Unidos. Posteriormente, el Instituto de Vigilancia de Medicamentos y Alimentos (INVIMA) de Colombia expidió registro sanitario para nivolumab adyuvante.

El KEYNOTE-054 es un estudio placebo controlado en el que se incluyeron 1.019 pacientes con melanoma etapa IIIA de alto riesgo IIIB, IIIC y IIID, de la nueva clasificación, a los que se les realizó resección completa, incluyendo disección ganglionar regional en las 13 semanas 
previas a la inclusión. Se excluyeron pacientes con metástasis ganglionar de $\leq 1 \mathrm{~mm}{ }^{17}$. Los criterios de exclusión fueron: metástasis en tránsito, infecciones no controladas, historia de enfermedad autoinmune, requerimiento de uso continuo de glucocorticoide sistémico, y antecedente de terapia sistémica previa para el melanoma. El brazo experimental consistió en pembrolizumab $200 \mathrm{mg}$ intravenoso cada tres semanas, por 18 dosis. La supervivencia libre de recaída a los 12 meses en el grupo que recibió pembrolizumab fue de $75.4 \%$, comparada con $61.0 \%$ en el grupo placebo ${ }^{17}$. Al igual que en el CheckMate-238, el beneficio se observó a través de todos los niveles de expresión en las células tumorales de PD-L1. El beneficio también se observó en pacientes con mutación del BRAF. A diferencia del estudio con nivolumab, el estudio con pembrolizumab incluyó pacientes con etapas IIIA. La supervivencia libre de recaída a los 18 meses con pembrolizumab fue superior en este subgrupo de pacientes con $89 \%$, comparada con $76.8 \%$.

Los eventos adversos grado $3 / 4$ con pembrolizumab ocurrieron en $31.6 \%$ de los pacientes, y el $13 \%$ suspendió el tratamiento por toxicidad ${ }^{17}$.

En febrero 15 de 2019, se aprobó nivolumab por la FDA en los Estados Unidos. En Colombia, el pembrolizumab no tiene registro sanitario para terapia adyuvante en melanoma al momento de elaboración de este documento.

\section{Terapia dirigida}

Se ha establecido que la mutación del BRAF en la posición V600 constituye un poderoso conductor que fomenta el fenotipo oncológico en melanoma. También se ha demostrado beneficio en melanoma avanzado BRAF mutado con el uso de agentes anti-BRAF solos, o con la inhibición dual de la vía de señalización con la coadministración de agentes anti-MEK ${ }^{18}$.

En el estudio COMBI-AD se incluyeron 870 pacientes con melanoma cutáneo etapas III resecados (también disección ganglionar regional), con mutación de BRAF V600E o V600K 19. Se incluyeron pacientes $\geq 18$ años de edad, melanoma cutáneo etapa III (en etapa IIIA se requería micrometástasis de $>1 \mathrm{~mm}$ de diámetro mayor, y se excluyeron las metástasis en tránsito), además de extirpación completa con linfadenectomía regional completa o resección, 12 semanas antes de la aleatorización, con desempeño ECOG o/1. El brazo experimental recibió un año de dabrafenib (un inhibidor oral de BRAF) 150 miligramos vía oral, dos veces al día, y trametinib (un agente anti-MEK), 2 mg vía oral cada día. El brazo control recibió placebos ${ }^{19}$.

Luego de un seguimiento mediano de 2.8 años, la supervivencia libre de recaída fue superior con dabrafenib/trametinib que la del placebo (67\% comparado con $44 \%$, respectivamente), con un cociente de riesgo de 0.47. La supervivencia libre de metástasis a distancia también fue superior con dabrafenib/trametinib (cociente de riesgo de 0.51$)^{19}$. Los pacientes con etapas IIIA derivaron el mismo beneficio que los pacientes con enfermedad más avanzada.

El beneficio de supervivencia libre de recaída se mantuvo luego de 44 meses de seguimiento mediano ${ }^{20} \mathrm{y}$ utilizando el modelo mixto de tasa de curación de Weibull, se postula una tasa de curación del 54 \% en el grupo tratado con dabrafenib/trametinib, comparado con $37 \%$ en el grupo placebo.

La terapia con dabrafenib y trametinib se asoció a eventos adversos grado 3/4 en el $41 \%$ de los pacientes. Los más comunes fueron pirexia, fatiga, náuseas, cefalea, escalofríos y diarrea. El tratamiento fue interrumpido en $66 \%$ de los pacientes y la dosis se redujo en $38 \%$. La descontinuación de tratamiento por eventos adversos ocurrió en $26 \%$ de los pacientes ${ }^{20}$.

\section{Impacto del manejo de ganglios re- gionales en la aplicación de los estu- dios de terapia adyuvante}

Como se ha explicado, todos los estudios de terapia sistémica adyuvante en melanoma etapa III requerían la disección ganglionar regional como parte de sus criterios de inclusión. Pero la evolución de la investigación clínica en los aspectos quirúrgicos del manejo de melanoma 
etapa III también ha sucedido en forma paralela y, el estándar de manejo actual, no requiere de la realización de disección ganglionar regional en todos los pacientes con melanoma con ganglio centinela positivo, al no impactar la supervivencia general ${ }^{21,22}$. Es aparente que el criterio de inclusión que exige la realización de disección ganglionar regional no va a ser aplicable a una proporción importante de pacientes con melanoma etapa III. En la opinión de los autores, esta dificultad es más teórica que real, al proceder con terapia sistémica adyuvante en los pacientes con etapa III que hayan completado el tratamiento locorregional requerido, y que sean candidatos a terapia adyuvante (especialmente, si se documenta metástasis ganglionar $>1 \mathrm{~mm}$ en su diámetro mayor, en el ganglio centinela).

\section{Comentario final}

La terapia sistémica adyuvante ha demostrado mejorar la supervivencia libre de recaída en melanoma cutáneo de alto riesgo resecado, definido como compromiso significativo de los ganglios linfáticos regionales o metástasis. Los estudios no fueron diseñados para mostrar beneficios en la supervivencia general, y no la han demostrado hasta la fecha. Sin embargo, se continúa el seguimiento de estas cohortes, y es posible que se tenga mayor claridad en el futuro sobre el impacto de estas tecnologías en la supervivencia general.

Las opciones de terapia adyuvante disponibles en Colombia se circunscriben a la combinación de dabrafenib más trametinib, para pacientes con mutación BRAF V600, o nivolumab, ambas por un año. Para pacientes con mutación BRAF etapa IIIA candidato a adyuvancia, se recomienda la combinación dabrafenib más trametinib, pues fue incluida en el estudio clínico de referencia. De igual forma, son candidatos a terapia anti BRAF/MEK el mismo grupo de pacientes con enfermedad autoinmune no endocrina o pacientes que requieran esteroides sis- témicos. Para pacientes con la misma mutación etapa IV resecada, se recomienda nivolumab, pues fueron incluidos en el estudio de referencia. Los autores conceptúan que nivolumab es también una alternativa razonable en Colombia para terapia adyuvante en etapa IIIA sin mutación BRAF, pues existe evidencia contundente de su beneficio en estudios con terapia anti-PD1. En otros escenarios en los que las indicaciones de ambas estrategias sean viables, se debe aplicar el juicio clínico, así como la consideración de otras variables como conveniencia, acceso y costo.

\section{Conclusión}

En conclusión, para pacientes con melanoma de alto riesgo resecados se recomienda tratamiento adyuvante sistémico con agentes anti PD1 como nivolumab o pembrolizumab. También es una opción la terapia dirigida con dabrafenib más trametinib adyuvante en pacientes con mutación de BRAF. La selección de la modalidad a practicar depende de variables clínicas y biológicas que se deciden en la interacción médico-paciente.

- $\quad$ Fuente de financiación: Los autores no declaran fuente de financiación.

- Conflicto de interés: Los autores declaran las siguientes relaciones que podrían generar sesgo. Mauricio Lema Medina: LILLY (conferencias), BMS (conferencias, consultorías, becas de investigación), MSD (becas de investigación), ROCHE (conferencias, consultorías, becas de investigación), NOVARTIS (confererencias), AZTRA-ZENECA (conferencias, consultoría), BOEHRINGER-INGELHEIM (conferencias, consultoría), PFIZER (conferencias, becas de investigación), ABBVIE (conferencias), AMAREY (conferencias, becas de investigación). Camila Lema Calidonio declara no tener ningún conflincto de interés. 


\section{Referencias}

1. Gershenwald JE, Scolyer RA, Hess KR, Sondak VK, Long $\mathrm{G}$ V., et al. Melanoma staging: Evidence-based changes in the American Joint Committee on Cancer eighth edition cancer staging manual. CA Cancer J [Internet]. Clin. 2017;67(6):472-92. https://doi.org/10.3322/caac.21409

2. Kirkwood JM, Strawderman MH, Ernstoff MS, Smith TJ, Borden EC, Blum RH. Interferon alfa-2b adjuvant therapy of high-risk resected cutaneous melanoma: The Eastern Cooperative Oncology Group trial EST 1684. J Clin Oncol [Internet]. 1996;14(1):7-17. https://doi.org/10.1200/ jco.1996.14.1.7

3. Kirkwood JM, Manola J, Ibrahim J, Sondak V, Ernstoff MS, Rao U. A Pooled Analysis of Eastern Cooperative Oncology Group and Intergroup Trials of Adjuvant High-Dose Interferon for Melanoma. Clin Cancer Res [Internet]. 2004;10(5):1670-7. https://doi.org/10.1158/1078-0432.ccr-1103-3

4. Kirkwood JM, Ibrahim JG, Sondak VK, Richards J, Flaherty LE, Ernstoff MS, et al. High- and low-dose interferon alfa-2b in high-risk melanoma: First analysis of intergroup trial E1690/S9111/C9190. J Clin Oncol [Internet]. 2000;18(12):2444-58. https://doi.org/10.1200/jco.2000.18.12.2444

5. Kirkwood JM, Ibrahim JG, Sosman JA, Sondak VK, Agarwala SS, Ernstoff MS, et al. High-dose interferon alfa-2b significantly prolongs relapse-free and overall survival compared with the GM2-KLH/QS-21 vaccine in patients with resected stage IIB-III melanoma: Results of intergroup trial E1694/S9512/C509801. J Clin Oncol [Internet]. 2001;19(9):2370-80. https://doi.org/10.1200/ jco.2001.19.9.2370

6. Eggermont AM, Suciu S, Santinami M, Testori A, Kruit WH, Marsden J, et al. Adjuvant therapy with pegylated interferon alfa-2b versus observation alone in resected stage III melanoma: final results of EORTC 18991, a randomised phase III trial. Lancet [Internet]. 2008;372(9633):117-26. https://doi. org/10.1016/s0140-6736(08)61033-8

7. Eggermont $A M M$, Suciu S, Testori A, Santinami M, Kruit WHJ, Marsden J, et al. Long-term results of the randomized phase III trial EORTC 18991 of adjuvant therapy with pegylated interferon alfa-2b versus observation in resected stage III melanoma. J Clin Oncol [Internet]. 2012;30(31):3810-8. https://doi.org/10.1200/jco.2011.41.3799

8. Mocellin S, Lens MB, Pasquali S, Pilati P, Chiarion Sileni V. Interferon alpha for the adjuvant treatment of cutaneous melanoma. Cochrane Databa- se Syst Rev [Internet]. 2013;2013(6). https://doi. org/10.1002/14651858.cdoo8955.pub2

9. Najjar YG, Puligandla M, Lee SJ, Kirkwood JM. An updated analysis of 4 randomized ECOG trials of high-dose interferon in the adjuvant treatment of melanoma. Cancer [Internet]. 2019;125(17):301324. https://doi.org/10.1002/cncr.32162

10. Hodi FS, O`Day SJ, McDermott DF, Weber RW, Sosman JA, Haanen JB, et al. Improved Survival with Ipilimumab in Patients with Metastatic Melanoma. Ne [Internet]. 2010;363:711-23. https://doi. org/10.1056/nejmoa1003466

11. Eggermont AMM, Chiarion-sileni V, Grob JJ, Dummer R, Wolchok JD, Schmidt $\mathrm{H}$, et al. Prolonged survival in stage III melanoma with ipilimumab adjuvant therapy. N Engl J Med [Internet]. 2016;375(19):1845-55. https://doi.org/10.1056/NEJMoa1611299

12. Tarhini AA, Lee SJ, Hodi FS, Rao UNM, Cohen $\mathrm{GI}$, Hamid O, et al. A phase III randomized study of adjuvant ipilimumab ( 3 or $10 \mathrm{mg} / \mathrm{kg}$ ) versus high-dose interferon alfa-2b for resected high-risk melanoma (U.S. Intergroup E1609): Preliminary safety and efficacy of the ipilimumab arms. J Clin Oncol [Internet]. 2017;35(15_suppl, 9500-9500). https://doi.org/10.1200/jco.19.01381

13. Robert C, Long GV, Brady B, Dutriaux C, Maio M, Mortier L, et al. Nivolumab in Previously Untreated Melanoma without BRAF Mutation. N Engl J Med [Internet]. 2014;372:320-30. https://doi. org/10.1056/nejmoa1412082

14. Hellmann MD, Paz-Ares L, Bernabe Caro R, Zurawski $\mathrm{B}$, Kim SW, Carcereny Costa $\mathrm{E}$, et al. Nivolumab plus Ipilimumab in Advanced Non-SmaII-Cell Lung Cancer. N Engl J Med [Internet]. 2019;381(21):2020-31. https://doi.org/10.1056/nejmoa1910231

15. Ascierto PA, Del Vecchio $M$, Mandalá $M$, Gogas $\mathrm{H}$, Arance AM, Dalle S, et al. Adjuvant Nivolumab versus Ipilimumab in Resected Stage III or IV Melanoma. N Engl J Med [Internet]. 2017;377(19):182435. https://doi.org/10.1016/s1470-2045(20)30494-0 16. Weber JS, Vecchio M Del, Mandala M, Gogas H, Arance AM, Dalle $S$, et al. Melanoma and other skin tumours. Ann Oncol [Internet]. 2019;30(Supplement 5):533-4.

17. Eggermont A, Blank C, Mandala M, Long G, Atkinson V, Dalle $S$, et al. Adjuvant Pembrolizumab versus Placebo in Resected Stage III Melanoma. N Engl J Med [Internet]. 2018;378(19):1789-801. https://doi.org/10.1056/NEJMoa1802357

18. Robert C, Grob J, Stroyakovskiy D, Karaszewska B, Hauschild A, Levchenko E, et al. Five-Year 
Outcomes with Dabrafenib plus Trametinib in Metastatic Melanoma. N Engl J Med [Internet]. 2019;381(7):626-36. https://doi.org/10.1056/nejmoa1904059

19. Long $G$ V., Hauschild A, Santinami M, Atkinson V, Mandalà $M$, Chiarion-Sileni V, et al. Adjuvant Dabrafenib plus Trametinib in Stage III BRAF-Mutated Melanoma. N Engl J Med [Internet]. 2017;377:1813-23. https://doi.org/10.1056/NEJMoa1708539

20. Hauschild A, Dummer R, Schadendorf D, Santinami $M$, Atkinson $V$, Mandal $M$, et al. Longer Follow-Up Confirms Relapse-Free Survival Benefit With Adjuvant Dabrafenib Plus Trametinib in Patients With Resected BRAF V600-Mutant Stage III Melanoma. J Clin Oncol [Internet].
2018;36(35):3441-9.

https://doi.org/10.1200/ jco.18.01219

21. Leiter U, Stadler R, Mauch C, Hohenberger W, Brockmeyer N, Berking C, et al. Complete lymph node dissection versus no dissection in patients with sentinel lymph node biopsy positive melanoma ( DeCOG-SLT ): a multicentre, randomised, phase 3 trial. Lancet Oncol [Internet]. 2016;2045(16):1-11. https://doi.org/10.1016/s14702045(16)00141-8

22. Faries $M$, Thompson J, Cochran A, Andtbacka R, Mozzillo N, Zager J, et al. Completion Dissection or Observation for Sentinel-Node Metastasis in Melanoma. N Engl J Med [Internet]. 2017;376(23):2211-22. https://doi.org/10.1056/nejmoa1613210 